\title{
DISCUSSION
}

\section{Two large-scale injection experiments and assessment of the advection- dispersion-filtration model}

\author{
F. BOUCHELAGHEM (2002). Géotechnique 52, No. 9, 667-682
}

Y.S. Kim \& A.J. Whittle, Massachusetts Institute of Technology, USA

We have read with great interest the Author's theoretical modelling and experimental validation of the flow and transport of microfine cement grout in soil in this paper, and in three other related publications (Bouchelaghem \& Vulliet, 2001; Bouchelaghem et al., 2001; Bouchelaghem \& Almosni, 2003). We have also tried to replicate the author's analyses through our own numerical simulations, and have found a number of issues that deserve further clarification regarding the interpretation of experimental data, as well as some inconsistencies in the selection of input parameters, such as the value of the initial skeleton density, $\left(\rho_{\mathrm{s}}\right)_{0}$. The value given in the Géotechnique paper, $2662.6 \mathrm{~kg} / \mathrm{m}^{3}$, is different from the value $2150 \mathrm{~kg} / \mathrm{m}^{3}$ quoted in Bouchelaghem \& Vulliet (2001) and in Bouchelaghem et al. (2001), although the same types of grout and sand (Leman sand) were considered in all three papers.

The author argues that the heterogeneous nature of the grout filtration might explain the underestimation of the pore pressures by numerical analysis, because the applied filtration model was based on the Kozeny-Carman equation and a constant filtration rate that did not depict the clogging of the pores causing sudden increases in the pressure. Although we generally agree with the author's point on the importance of the modelling of clogging, clogging does not seem to be solely responsible for the discrepancies between numerical and experimental results. The experimental results in Fig. 5, for example, show noticeable flattening at the later part of the injection that the numerical simulations fail to predict, and they do not seem to be improved by including clogging in the model.

A more plausible explanation for stabilisation of the pore pressures is that the soil close to the injection point loses its capacity to act as a filter when all available filtration sites (i.e. locations where grout particles can be deposited) become saturated. Indeed, this concept of a limiting filtration capacity seems to be incorporated in the author's model using the maximum filtered grout density, $\left(\rho_{\sigma}\right)_{\max }$, which appears in the Kozeny-Carman equation (10) in Bouchelaghem \& Vulliet (2001):

$$
\begin{aligned}
& \frac{k}{k_{0}}=\left(\frac{n}{n_{0}}\right)^{3}\left(\frac{1-n_{0}}{1-n}\right)^{4 / 3}, \rho_{\sigma} \leqslant\left(\rho_{\sigma}\right)_{\max } \\
& =\left(1-\frac{\rho_{\sigma}}{n_{0} \bar{\rho}_{\mathrm{s}}^{\mathrm{s}}}\right)^{3}\left(1+\frac{\rho_{\sigma}}{\left(1-n_{0}\right) \bar{\rho}_{\mathrm{s}}^{\mathrm{s}}}\right)^{-4 / 3}
\end{aligned}
$$

where $k, k_{0}$ and $n, n_{0}$ are the current and initial values of the permeability and porosity respectively, $\left(\rho_{\sigma}\right)$ and $\left(\rho_{\sigma}\right)_{\max }$ are the filtered grout density and its maximum value, and $\bar{\rho}_{\mathrm{s}}^{\mathrm{s}}$ is the solid skeleton's intrinsic density. This equation can be rearranged as follows:

$$
n=n_{0}-\frac{\rho_{\sigma}}{\bar{\rho}_{\mathrm{s}}^{\mathrm{s}}}, n_{\min } \leqslant n
$$

where $n_{\min }$ denotes the minimum porosity corresponding to the maximum filtered grout density, $\left(\rho_{\sigma}\right)_{\max }$. There is a quote in the same paper that $\left(\rho_{\sigma}\right)_{\max }$ lies between $0 \cdot 2 n_{0} \bar{\rho}_{\mathrm{s}}^{\mathrm{s}}$ and $0.4 n_{0} \bar{\rho}_{\mathrm{s}}^{\mathrm{s}}$, which implies that the minimum porosity of the soil deposit is in the range $0 \cdot 6 n_{0} \leqslant n_{\min } \leqslant 0 \cdot 8 n_{0}$.

A set of numerical simulations using a finite-difference scheme was carried out to evaluate the effect of the minimum porosity on the pore pressure predictions. We have implemented the same formulation as presented by the author, with one exception. We have ignored deformations of the soil skeleton based on the measured data that show minimal displacements (less than $10 \mu \mathrm{m}$ ). Fig. 15 compares the results of our simulations for the pore fluid pressures at radius $r=0.045 \mathrm{~m}$ in the second large-scale test with an injection rate of $61 / \mathrm{min}$ with the experimental data reported in Fig. 12 (the author does not indicate an exact elevation of the monitoring point, but our simulations are shown at a point $0.71 \mathrm{~m}$ above the base of the chamber). Fig. 15 shows significant differences in the predicted pore pressures for $t$ $>1150 \mathrm{~s}$ between two simulations with $n_{\min }=0 \cdot 8 n_{0}$ and $0 \cdot 7 n_{0}$. For $n_{\min }=0 \cdot 8 n_{0}$ the minimum porosity is achieved at $t \approx 1150 \mathrm{~s}$, effectively arresting further increases in pore pressures, a behaviour that matches closely the measured data in this experiment. When a smaller limit on porosity is imposed $\left(n_{\min }=0 \cdot 7 n_{0}\right)$, pore pressures continue to increase unabated for $t>1150 \mathrm{~s}$ at the same or even a higher rate. The author does not give details of the minimum porosity (or maximum filtered grout density) used in the original simulations, although the predictions given for $r=0.02 \mathrm{~m}$

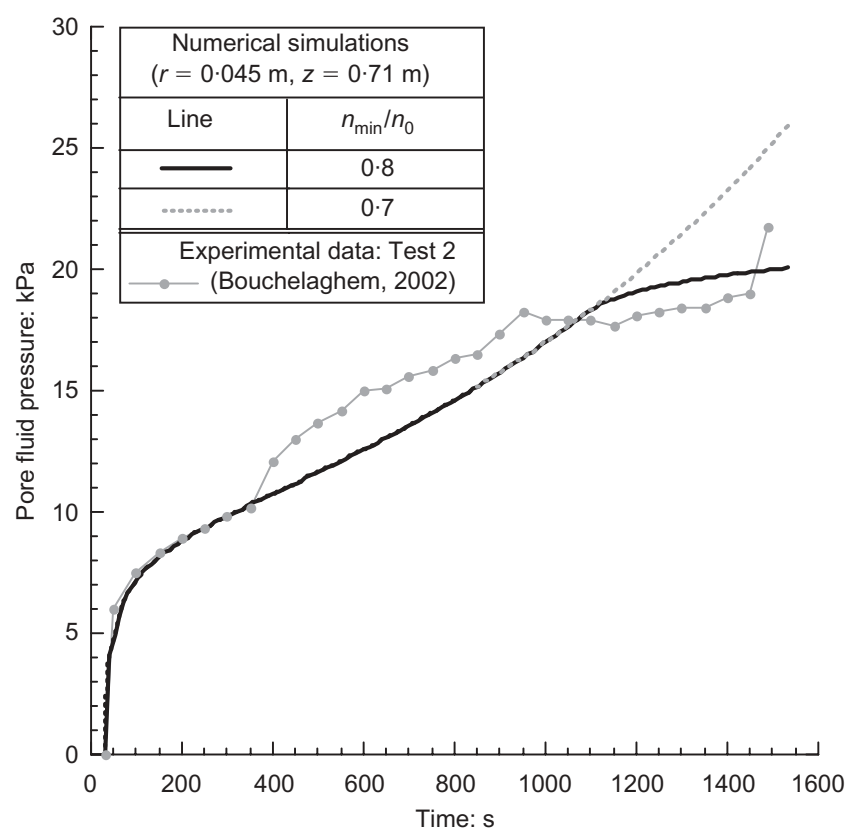

Fig. 15. Effects of a minimum porosity limit on predictions of pore fluid pressure for large-scale injection test 2 . 
in test 1 (Fig. 4) suggest that this limit is included in the simulations. It would be very helpful if the author could clarify the value(s) of $\left(\rho_{\sigma}\right)_{\max }$ used and the basis for selecting this parameter.

The predictions for injection test 2 with $n_{\min }=0.7 n_{0}$ should be directly comparable with the predictions presented by the author. However, Fig. 16 shows a clear discrepancy between our simulations of pore fluid pressure at $r=$ $0.045 \mathrm{~m}$ and those presented by the author in Fig. 12 based on the specified longitudinal dispersivity, $a_{\mathrm{L}}=10^{-3} \mathrm{~m}$, quoted in Table 1. The figure shows much better agreement when the longitudinal dispersivity is increased $\left(a_{\mathrm{L}}=3 \times\right.$ $10^{-2}$, and $10^{-2} \mathrm{~m}$ in Fig. 16). We find that these values of dispersivity are consistent with values quoted by Bouchelaghem \& Almosni (2003) for identical test materials as well as with the concentration profiles shown in Fig. 9.

The author recognises that the assumption of a constant filtration rate represents the main limitation in the theoretical model, and recommends that a more general expression for filtration rate $\lambda$ (equation (8)) should be specified with the help of microscopic information on deposition mechanisms. Although we agree that such information will be essential for a better model, we doubt whether it can be achieved by a simple filtration function. Processes of re-entrainment and clogging are highly dependent on details of the pore structure as well as on the deposit morphology. In contrast, equation (8) was derived through an averaging process applied to a unit-collector model (in which the filters are modelled as identical spheres) representing regular deposition on a clean filter. In our opinion, the development of realistic microscopic models for the filtration mechanisms together with the formulation of suitable up-scaling relations are the more critical and challenging tasks for advancing model predictive capabilities.

\section{NOTATION}

$k$ intrinsic permeability

$n$ porosity

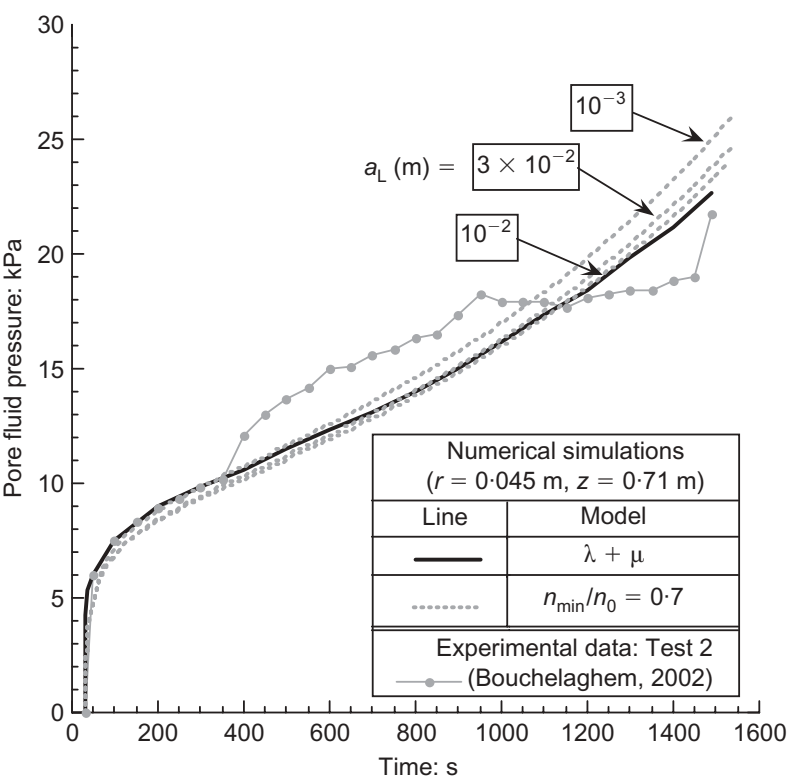

Fig. 16. Comparison of predictions for pore fluid pressure for large-scale injection test 2 with results presented by author

$\rho_{\sigma} \quad$ filtered grout density

$\bar{\rho}_{\mathrm{s}}^{\mathrm{s}}$ intrinsic density of solid skeleton

$a_{\mathrm{L}}$ longitudinal dispersivity constant

\section{REFERENCE}

Bouchelaghem, F. \& Almosni, A. (2003). Experimental determination of the longitudinal dispersivity during the injection of a micro-cement grout in a one-dimensional soil column. Transport in Porous Media, 52, No. 1, 67-94. 